Journal of Case Reports 2018;8(3):202-204

\title{
Autoimmune Hepatitis: The Third Autoimmune Disease in a Young Female
}

\author{
Rasangi Sumudu Clare Suraweera, Srivickneswaran Ghetheeswaran, Jansan Jesuthasan \\ Department of Medicine, Jaffna Teaching Hospital, Jaffna, Srilanka.
}

Corresponding Author:

Dr. Rasangi Sumudu Clare Suraweera

Email: ras_clare@hotmail.com

This is an Open Access article distributed under the terms of the Creative Commons Attribution License (creativecommons.org/ licenses/by/3.0).

Received

Accepted

February 22, 2018

Published

July 13,2018

August 25, 2018

\begin{abstract}
Background: Deranged liver functions in patients with systemic lupus erythematosus (SLE) may be caused by drugs, viral hepatitis, fatty liver or lupus hepatitis; however SLE patient with autoimmune thyroiditis developing autoimmune hepatitis have rarely been described. Case Report: A 22 year old girl who had a background history of systemic lupus erythematosus and autoimmune thyroiditis presented to us with altered liver functions and right upper quadrant pain and was diagnosed to have autoimmune hepatitis. Conclusion: Autoimmune hepatitis is a rare in patients with SLE but polyautoimmunity is something we need to consider in patients who already have one existing autoimmune disease. Polyautoimmunity should be added in list of differential diagnosis when investigating patients with deranged liver functions if the clinical picture is suggestive.
\end{abstract}

Keywords: Autoimmune Hepatitis, Autoimmune Thyroiditis, Pain, Systemic Lupus Erythematosus.

\section{Introduction}

Autoimmune hepatitis (AIH), chronic progressive disease is characterized by immune mediated destruction of the liver parenchyma and the presence of autoantibodies in the periphery. It predominantly affects women and its sequale are cirrhosis, liver failure and death [1]. Autoimmune hepatitis at times might coexist with other diseases such as hemolytic anemia, thyroiditis, immune thrombocytopenia, rheumatoid arthritis and systemic lupus erythematous [2].

\section{Case Report}

Our case is 22 year old lady who was being followed in our medical clinic for systemic lupus erythematosus and autoimmune thyroiditis. She presented with right upper abdominal pain of four months duration. She complained of nausea periodically and also had some loss of appetite. She gave no history suggestive of jaundice, yellowish discolouration of the eyes or pale coloured stools. There was no history of blood transfusions, tattooing or consumption of ayruvedic medication.
Initially she was treated for dyspepsia for about one month and but her symptoms failed to resolve. Her liver function tests were done which revealed an aspartate aminotransferase $(\mathrm{AST})=276 \mathrm{U} / \mathrm{L}$ $(15-37)$ and alanine aminotransferase $($ ALT $)=381$ U/L (16-63); the liver functions were repeated a month later and had not changed significantly $\mathrm{AST}=231 \mathrm{U} / \mathrm{L}$ and $\mathrm{ALT}=300 \mathrm{U} / \mathrm{L}$. The patient was taking azathioprine and hydroxycholoroquine for her systemic lupus erythematosus (SLE) and thyroxine. Since she had not suffered any flares of SLE and her ESR was normal, her azathioprine was stopped. We attributed the derangement of her liver functions to the usage of azathioprine but they did not improve significantly after stopping the drug and the patient was still symptomatic. At this point we considered the possibility of her having autoimmune hepatitis as poly-autoimmuntiy is a quite a prevalent entity. She was admitted and her liver functions, viral markers and immunoglobulin $\mathrm{G}$ levels were sent. The investigations revealed alkaline phosphatase $=101 \mathrm{U} / \mathrm{L}$ (46-116), total protein $=98 \mathrm{~g} / \mathrm{L}$ (64-82), albumin= $34 \mathrm{~g} / \mathrm{L}$ (34-50), globulin= $64 \mathrm{~g} / \mathrm{L}(22-48)$, total bilirubin $=18.8$ 
$\mathrm{mmol} / \mathrm{L}(0-17.1)$, direct bilirubin= $3.4 \mathrm{mmol} / \mathrm{L}$ (0-3). The serological markers were positive antinuclear antibody in 1:160 dilution, negative antismooth muscle antibody, $\operatorname{IgG}=3000$ (569-1919), and negative hepatitis $\mathrm{C}$ antibody and hepatitis $\mathrm{B}$ surface antigen. Her abdominal ultrasound showed liver cirrhosis with fibrosis, portal tracts were mildly enlarged and no para-aortic lymph-node enlargement was noted. The elevated IgG prompted us to do the liver biopsy. The liver biopsy showed parenchymal tissue with effaced architecture bridging fibrosis and nodule formation. The portal tracts were predominantly lympho-plasmocytic. There was moderate interface reaction. According to the International autoimmune hepatitis score she had a total score of seven where her ANA was positive more than 1:80 and her IgG levels were more than 1.10 times normal, there were features typical of autoimmune hepatitis in the liver biopsy and the presence of viral hepatitis was excluded [4]. No abnormality was noted on upper GI endoscopy.

While she was being investigated the patient developed a flare up of her SLE as her azathioprine and hydroxy-cholorquine had been stopped. She was restarted on hydroxy-choroquine $200 \mathrm{mg}$ daily after an ophthalmology checkup to rule out maculopathy and prednioslone $40 \mathrm{mg}$ daily with tapering of the dose. We started her on azathioprine at $50 \mathrm{mg}$ daily when the prednisolone dose was reduced to $20 \mathrm{mg}$. The prednisolone has been tapered off and the patients liver functions have reduced dramatically.

\section{Discussion}

Our patient had features of polyautoimmunity which is the presence of two or more autoimmune diseases in a single patient [7]. She had autoimmune thyroiditis, SLE and autoimmune hepatitis. Although SLE affects various organ systems, liver involvement is usually not part of the disease spectrum $[3,4]$. The reasons of deranged liver enzymes in SLE patients are hepatotoxic drugs, viral hepatitis, fatty liver disease associated with steroid use and less commonly primary liver disease, hepatic congestion or lupus hepatitis [2]. It is quite rare to find an autoimmune hepatitis and SLE overlap and is usually considered when patients fulfill the American College of Rheumatology criteria for systemic lupus erythematous and International autoimmune hepatitis group scoring for AIH [5].

Distinguishing between autoimmune hepatitis and systemic lupus erythematosus can be tough as quite a number of the auto-antibodies are duplicated in the two entities. Immunoglobulin $\mathrm{G}$ and anti-nuclear antibodies are present in both $\mathrm{AIH}$ and SLE and there are few markers that help distinguish AIH from SLE such as soluble liver antigen (SLA), liver-pancreas, smooth-muscle antibody (SMA) with specificity for F-actin and microsomal autoantigen; such as anti-liver kidney mitochondrial antibodies (anti-LKM antibody) are present in AIH and not SLE [3]. Due to this lack of a specific serological marker in autoimmune hepatitis; a liver biopsy is considered mandatory.

Anti-ribosomal $\mathrm{P}$ antibody has been suggested to be useful in differentiating SLE associated hepatitis from AIH. Anti-Sm (antiSmith) antibodies are very specific for SLE but not sensitive [5]. Hyper-gammaglobulinemia is a characteristic of autoimmune hepatitis and Borberg et al. showed that a higher mortality was noted in patients with autoimmune hepatitis showing a two fold increase in serum IgG levels and associated sustained raise in ALT levels [6]. Doo Ho Lim et al. showed that patients with SLE - AIH overlap and have much higher levels of IgG than those with primary autoimmune hepatitis and that those with SLE - AIH patients who had cirrhosis had higher levels of IgG than those without cirrhosis. They also noticed that the serum IgG levels normalized in patients with SLE-AIH without cirrhosis after treatment suggesting that $\mathrm{IgG}$ can be used as a marker for prognosis in SLE-AIH [2]. 


\section{Conclusion}

Autoimmune hepatitis is a rare event in patients with SLE but polyautoimmunity is something we need to consider in patients who already have one existing autoimmune disease. Autoimmune hepatitis should be considered as differential in patients with deranged liver functions if the clinical picture is suggestive.

Contributors: RSCS: manuscript writing, patient management; SG: manuscript editing, patient management; JJ: critical inputs into the manuscript. RSCS will act as guarantor. All authors approved the final version of this manuscript.

Funding: None; Competing interests: None stated.

\section{References}

1. Lammert C, Loy VM, Oshima K, Gawrieh S. Management of difficult cases of autoimmune hepatitis. Curr Gastroenterology. 2016;18:9.
2. HoLim D, Kim YG, Lee DB, Ahn SM, Hong S, Lee CK, Bin Yoo. Immunoglobulin $\mathrm{G}$ levels as a prognostic factor for autoimmune hepatitis combined with systemic lupus erythematosus arthritis care and research. American College of Rheumatology. 2016;68:995-1002.

3. Beisel C, Weiler-Normann C, Teufel A, Lohse AW. Association of autoimmune hepatitis and systemic lupus erythematosus: a case series and review of literature. World J Gastroenterol. 2014;20:12662-12667.

4. Gleeson D, Heneghan MA. British Society of Gastroenterology (BSG) guidelines for management of autoimmune hepatitis. Gut 2011;60:1611-1629.

5. Koshy JM, John M. Autoimmune Hepatitis -SLE overlap syndrome. J Assoc Physicians India. 2012;60:59-60.

6. Boberg KM, Aadland E, Jahnsen J, Raknerud N, Stiris $\mathrm{M}$, Bell $\mathrm{H}$. Incidence and prevalence of primary biliary cirrhosis, primary sclerosing cholangitis, and autoimmune hepatitis in a Norwegian population. Scand J Gastroenterol. 1998;33:99-103. 